\title{
L'enseignement de la langue régionale en Alsace et en Moselle
}

Daniel Morgen et Armand Zimmer

\section{(2) OpenEdition \\ Journals}

Édition électronique

URL : http://journals.openedition.org/trema/981

DOI : 10.4000/trema.981

ISSN : 2107-0997

Éditeur

Faculté d'Éducation de l'université de Montpellier

Édition imprimée

Date de publication : 1 septembre 2009

Pagination : 109-118

ISSN : 1167-315X

Référence électronique

Daniel Morgen et Armand Zimmer, "L'enseignement de la langue régionale en Alsace et en Moselle », Tréma [En ligne], 31 | 2009, mis en ligne le 01 septembre 2011, consulté le 19 avril 2019. URL : http:// journals.openedition.org/trema/981; DOI : 10.4000/trema.981

Ce document a été généré automatiquement le 19 avril 2019

Trema 


\title{
L'enseignement de la langue régionale en Alsace et en Moselle
}

\author{
Daniel Morgen et Armand Zimmer
}

En Alsace et en Moselle, la langue régionale existe sous deux formes: les dialectes alémaniques et franciques parlés en Alsace et en Moselle, dialectes de l'allemand, d'une part, l'allemand standard d'autre part. Celui - ci est la langue de référence des dialectes (Ministère de l'Éducation nationale, arrêté du 26 décembre 2007, B.O. $\mathrm{n}^{\circ} 3$, janvier 2008). Avant de présenter la situation actuelle dans ses paramètres statistiques et structurels ainsi que le développement des enseignements, il convient de revenir sur la définition de la langue régionale donnée ci - dessus pour en présenter les contours et les enjeux pédagogiques.

\section{La langue régionale} d'Alsace et des Pays mosellans

Les variétés dialectales parlées en Alsace et en Moselle thioise appartiennent à la famille des langues germaniques, au même titre que d'autres dialectes allemands. Mais à la différence des dialectes franciques et alémaniques parlés dans les régions germanophones voisines, les dialectes alsaciens et mosellans sont aujourd'hui, dans une certaine mesure, coupés de leur langue de référence, l'allemand standard. Leur déclin est certes lié aux reculs de la pratique et de la transmission, mais aussi à la distance de plus en plus grande par rapport à l'allemand qui évolue de son côté. Or, nos dialectes ont 
besoin de l'allemand pour se ressourcer, pour acquérir des mots nouveaux ou pour dire les réalités nouvelles. Faute de quoi, aucune communication durable et continue ne sera possible dans ces variétés linguistiques. Langues de l'amitié, du vécu quotidien, les dialectes alsaciens et mosellans échappent à la modernité et régressent. Ils régressent d'autant plus que les seuls emprunts sont faits au français, et que les emprunts, jusqu'ici surtout lexicaux, ont tendance à influencer aussi la syntaxe de la langue. Quel avenir pour les dialectes sans l'allemand? Sans code écrit, sans grammaire institutionnalisée, ils ne s'enseignent pas comme on enseigne une langue codifiée et ne peuvent guère servir de langue d'enseignement en dehors de l'oral. Dès qu'intervient l'écrit, la langue instrumentale est l'allemand standard, qui dispose d'un code attesté. Pour l'enseignement, la conclusion doit être celle - ci : le dialecte alsacien ou mosellan pour l'oral ou pour une partie de l'oral, l'allemand standard pour l'oral et pour l'écrit. Mais tout enfant, tout adolescent doit être informé de l'existence de ces dialectes, initié à ses aspects spécifiques et à ses richesses littéraires ou autres. Ce n'est donc pas un enseignement, mais une pratique des dialectes au sein de l'école que l'on doit envisager.

Quelle que soit la période concernée, la langue régionale a toujours été enseignée sous sa forme standard, la (deutsche) Hochsprache, comme un fait qui allait de soi. Ainsi, l'allemand a été présent jusqu'à six heures par semaine entre 1920 et 1940 dans l'enseignement primaire et primaire supérieur, formant

«des générations d'enfants bilingues, aux compétences sans doute inégales, mais qui, sous l'effet du plus grand désir d'ascension sociale et du contact plus fréquent avec l'écrit, acquièrent, beaucoup plus fréquemment que par le passé, des compétences élevées de lecteurs dans les deux langues » (HUCK, 2006, p. 338).

À cause de la deuxième guerre mondiale, cette situation de paix linguistique évolue brutalement : à la Libération, bien des Alsaciens et des Mosellans ont du mal à faire la différence entre la grande langue de culture, qui est aussi la première langue parlée sur le continent européen, et la langue de l'occupant national - socialiste. En même temps, les pouvoirs publics tentent de marginaliser le dialecte " parce qu'il représente [...] un obstacle central à la diffusion et à la pratique du français ... » mais «'d'un point de vue politique global, [l'État] estime l'usage du dialecte moins préjudiciable que celui de l'allemand standard. Il s'agit d'une politique linguistique stratégique, non pour promouvoir le dialecte, mais pour écarter l'allemand » (HUCK, 2008, p. 352, note 9).

5 La politique linguistique explicite est une politique prioritaire en faveur du français, avec une préférence avérée pour le français sur le dialecte et secondairement pour le dialecte sur l'allemand.

6 La loi DEIXONNE, abolie depuis 2005, ne s'applique pas à l'Alsace ni à la Moselle. Elle est partiellement compensée par un décret de décembre 1952 qui instaure l'enseignement de l'allemand dans les classes de fin d'études. Cette mesure se heurtera à l'opposition systématique du syndicat majoritaire, le S.N.I., mais, vaille que vaille, fonctionnera jusque dans les années soixante. Les rebonds de l'allemand s'appellent ensuite «La Réforme Holderith » en 1972, dont le succès est réel, sans doute parce que son initiateur avait réussi à s'assurer la neutralité des syndicats, puis le programme langue et culture régionales, grâce à la circulaire Savary de juin 1982 avec, dans les deux cas, une réintroduction de l'allemand dans les cours moyens puis au CE2. Après 1982, des textes 
rectoraux officialisent le statut, le rôle et la place de l'allemand par rapport aux parlers alsaciens et mosellans, développent une politique linguistique grandeur nature de l'école élémentaire au lycée et créent le socle de l'enseignement de la langue. Mais ces textes rectoraux écartent le concept éducatif de bilinguisme, ou alors ne l'acceptent que dans un sens restreint pour désigner la politique d'un enseignement de l'allemand à dose homéopathique ( 2 - 3 h/semaine). Il faudra donc attendre 1991 - 1992, avec l'arrivée du recteur Jean - Paul DE GAUDEMAR, pour voir se réaliser en Alsace la mise en œuvre d'une politique qui, avec réalisme et sans préjugés, considère que seule une politique volontariste d'immersion linguistique - où la langue n'est plus un simple objet mais un outil - peut combattre l'érosion de la langue régionale dans les compétences, et si possible, dans les pratiques. Si les politiques linguistiques en Alsace et en Moselle sont proches, chaque région tient à sa spécificité.

\section{La situation actuelle}

\section{1. Le socle d'enseignement et ses extensions}

7 Depuis près d'une vingtaine d'années, on considère, dans les sphères éducatives alsaciennes, que près de $95 \%$ des enfants du cycle 3 bénéficient d'un enseignement extensif de l'allemand, pour le distinguer, par cette épithète, de l'enseignement intensif, l'enseignement bilingue à parité horaire. Avec l'option « langue et culture régionales », cet enseignement constitue le socle du dispositif mis en place en grande partie entre 1982 et 1990 et détermine en grande partie la suite de l'apprentissage des langues : l'enfant qui a bénéficié de l'enseignement " précoce » de l'allemand à l'école primaire peut être admis dans des sections trilingues (actuelles sections bilangues), voire dans des sections européennes en allemand (cf. II.2.1).

8 Un programme ambitieux d'échanges régionaux de classes et d'élèves sous différentes formes, sublimé ces dernières années dans un programme de coopération éducative sur l'Internet ( $\mathrm{E}$ TWINING), ainsi que de nombreuses formes d'échanges individuels réciproques, de deux semaines à six mois, consolident le programme de langue. Dans l'enseignement professionnel, l'académie développe la « Mention régionale, connaissance de l'allemand en formation professionnelle ", créée vers 1985, en validant les stages dans une entreprise germanophone par le Certificat EUREGIO (EUREGIO - Pass). La Moselle connaît un dispositif analogue.

9 C'est sur ce socle "Langue et culture régionales" que se sont développés les sites bilingues en Alsace et biculturels, en Moselle.

\section{2. Le dispositif bilingue et plurilingue}

10 Dans un premier temps, ce sont les sections européennes, instituées en août 1992, qui ont assuré, dans les collèges et les lycées, le développement d'une immersion partielle mais minimale ( 2 à 3 heures d'enseignement dans la langue).

\section{2. 1. Les sections européennes}

11 Trente - sept sections ${ }^{1}$ européennes en allemand fonctionnent actuellement dans l'académie de Strasbourg. 
Dans leur grande majorité, ces sections appliquent les principes de la circulaire fondatrice d'août 1992 des sections européennes, conçues d'après le modèle allemand des «bilinguale Züge / sections ou voies bilingues »: démarrage au niveau de la classe de 4e par une intensification de l'enseignement de la langue, enseignement dans une discipline dite non linguistique à partir de la seconde. Mais, au lycée, trois ou quatre d'entre elles étendent ce principe à plus d'une discipline, jusqu'à trois ou quatre, assurant ainsi en partie le suivi de l'enseignement bilingue de collège. Nous y reviendrons.

\section{2. 2. L'enseignement bilingue précoce à parité horaire en Alsace}

Le développement du concept d'enseignement bilingue à parité horaire - qui, en grande partie, est un héritage du Pays basque recueilli lors de séjours sur place en 1991 et 1993 a été l'un des éléments les plus réussis de la période récente. Au bout d'une quinzaine d'années, cette forme d'enseignement s'est développée dans tout le système éducatif en prenant appui sur les principes fondateurs de précocité, de continuité, d'instrumentalisation et de parité des langues (GEIGER - JAILLET, MORGEN 2006). Elle a justifié la création d'une formation spécialisée d'enseignants dans le Centre de formation aux enseignements bilingues de Guebwiller (IUFM d'Alsace) et celui de Sarreguemines (IUFM de Lorraine). En 2007/2008, plus de 17000 élèves alsaciens bénéficiaient de cet enseignement.

\begin{tabular}{|c|c|c|c|c|c|}
\hline \multirow{2}{*}{$\begin{array}{l}2007 / 08 \\
\text { (Académie de Strasbourg) }\end{array}$} & \multirow{2}{*}{$\begin{array}{l}\text { Écoles } \\
\text { Collèges } \\
\text { Lycées }\end{array}$} & \multirow[t]{2}{*}{ classes } & \multicolumn{2}{|l|}{ élèves } & \multirow{2}{*}{$\begin{array}{l}\% \text { de } \\
\text { l'effectif } \\
\text { total }\end{array}$} \\
\hline & & & $\begin{array}{l}\text { Nb total } \\
2008 / 09\end{array}$ & & \\
\hline $\begin{array}{l}\text { Écoles maternelles } \\
\text { et élémentaires publiques ou } \\
\text { sous contrat }\end{array}$ & 250 & 635 & 181722 & 15618 & $8,6 \%$ \\
\hline Écoles ou classes hors contrat & & & $?$ & 874 & \\
\hline $\begin{array}{ll}\text { dont Écoles maternelles } & \text { et } \\
\text { élém entaires ABCM } & - \\
\text { Zweisprachigkeit } & \\
\text { Alsace (2008) } & \end{array}$ & 12 & 54 & & 818 & \\
\hline $\begin{array}{l}\text { Total élémentaire public et } \\
\text { privé }{ }^{2}\end{array}$ & & & & 16492 & \\
\hline $\begin{array}{l}\text { Collèges publics et privés sous } \\
\text { contrat } \\
\text { (nb total de collèges) }\end{array}$ & $\begin{array}{l}46 \\
(169)\end{array}$ & & 86671 & 2918 & env. $3,4 \%$ \\
\hline $\begin{array}{l}\text { Lycées publics (LEGT) et } \\
\text { privés sous contrat } \\
\text { Filière ABIBAC } \\
\text { (nb total de lycées) }\end{array}$ & $\begin{array}{l}12 \\
(101)\end{array}$ & & 40780 & 850 & $2 \%$ \\
\hline Total Alsace & & & & 20260 & \\
\hline
\end{tabular}

TAB 2 : les statistiques de l'enseignement bilingue en 2006/07 (Sources : Mission académique aux enseignements régionaux et internationaux, académie de Strasbourg, Office pour la langue et la culture d'Alsace (OLCA), ABCM - Zweisprachigkeit) et Marchal (2008). NB : les sections européennes ne figurent pas dans cette totalisation ${ }^{2}$

14 Depuis la rentrée 2008, tous les élèves des classes bilingues de sixième bénéficient de l'option langue et culture régionale qui était jusque là accessible seulement en option et à 
partir de la classe de quatrième. L'enseignement concerne les aspects historiques, linguistiques et culturels de la langue régionale qui sert aussi d'outil dans l'option. élémentaires et concernent un total de 2675 élèves. La scolarité se poursuit dans les 7 collèges de secteur. Les enseignants des sites biculturels sont habilités à enseigner l'allemand et la DNL.

17 Ces sites sont rattachés au dispositif mosellan d'enseignement approfondi de l'allemand (DEAA). Les élèves bénéficient d'un temps d'exposition à la langue allemande (avec une part importante de DNL) pouvant aller jusqu'à la parité horaire avec le français.

cie site bénéficie de la présence d'un maître allemand dans le cadre d'un programme d'échange d'enseignants, organisé par l'OFAJ. Cette disposition témoigne de l'effort exceptionnel consenti par l'inspection académique pour le développement du DEAA (10 ETP).

19 Les Erzieher(innen) sarroises intervenant dans le cadre d'un programme Interreg (III puis IV), le font en appui des équipes enseignantes en place. Ces éducateurs et éducatrices de jeunes enfants complètent le dispositif mais n'ont pas et n'ont jamais eu vocation de se substituer aux enseignants. Ils (elles) sont employé(e)s par les collectivités locales et formé(e)s pour la France par le Centre Transfrontalier.

L'antenne IUFM de Sarreguemines forme environ 25 PE chaque année, qui peuvent postuler un emploi dans les sites biculturels. L'Inspection Académique, quant à elle, programme chaque année plusieurs sessions de formation continue, dont des stages inter degrés, coordonnées par le Centre Transfrontalier de Saint - Avold, à destination des enseignants des sites biculturels. (ARDOUIN, 2009, p. 13). Comme son nom l'indique, ce «Centre de documentation et de formation pour l'apprentissage de la langue du voisin » a pour mission de promouvoir l'enseignement de la langue du voisin, de coordonner la formation continue des enseignants allemands et français ainsi que les échanges transfrontaliers, de produire et de fournir des outils pédagogiques. Il dispose d'une riche documentation sur l'enseignement de la langue ${ }^{5}$.

21 En dehors de ce dispositif public, l'association ABCM - Zweisprachigkeit gère deux sites bilingues dans la commune de Sarreguemines qui disposent chacun de deux classes maternelles et de quatre classes élémentaires (262 élèves). 


\section{Le partenariat Éducation nationale - Collectivités territoriales}

22 Le développement de l'enseignement de la langue régionale et celui de l'enseignement bilingue de et en langue régionale bénéficient, depuis une vingtaine d'années, du soutien des Collectivités territoriales alsaciennes. L'ensemble du dispositif - bilingue et non bilingue - repose sur des conventions entre l'État et les trois Collectivités territoriales. La plus récente d'entre elles a été signée le 13 juillet 2007 et porte sur la période 2007 - 2013. Pas assez volontariste aux yeux de certains qui suggèrent de faciliter l'accès à la titularisation d'étudiants allemands, trop aux yeux d'autres qui craignent que les viviers d'étudiants bilingues ne permettent pas d'atteindre des objectifs trop ambitieux, cette convention se fixe pour objectifs de consolider le programme académique, d'étendre le bénéfice de l'enseignement extensif à tous les élèves dès l'école maternelle et de doubler les effectifs accueillis dans l'enseignement bilingue (cf. tableau 2). La convention prévoit aussi la généralisation de l'anglais en deuxième langue dans toutes les classes de sixième. L'État prend à sa charge les enseignants. Les collectivités approvisionnent un fonds spécifique Langue et Culture régionales, qui finance en particulier, l'édition de manuels et d'outils pédagogiques. Mais, mise à part la convention entre l'inspection académique et le Conseil général de la Moselle au sujet du Centre transfrontalier, aucune convention de la même ampleur n'a été adoptée en Moselle.

\section{La production d'outils ${ }^{6}$}

Cependant, le financement des publications ne peut pas régler tous les problèmes. La rédaction des manuels et des outils avance à un rythme plus lent. Elle constitue un gros travail pour les enseignants qui s'y lancent. De plus, dans un contexte général de réduction des moyens, l'éducation nationale ne parvient pas à financer les décharges éventuelles. Enfin, le secteur des publications souffre de la taille réduite du public scolaire consommateur potentiel. De ce fait, aucun autre éditeur que le CRDP ne se lance dans un projet de production. Les équipes du premier degré, composées de jeunes enseignants pour la plupart, préfèrent entreprendre la traduction de manuels nationaux (Ex: Mathe mit Ermel, d'après les manuels ERMEL de l'IREM - Cap Maths CP à CE2) que de se lancer dans un projet original, plus périlleux.

En plus de ces deux outils de mathématiques, un manuel de géographie pour le cycle 3 et des albums de lecture pour différents niveaux ( $\mathrm{CP}, \mathrm{CM}$ ) sont disponibles, mais par contre, pour l'instant, aucun manuel de langue et grammaire dont l'urgence se fait sentir depuis longtemps. Un manuel de grammaire est annoncé pour 2009, mais il s'agit plutôt d'un précis de grammaire que d'un manuel d'enseignement. Le CRDP a privilégié la publication d'excellentes plaquettes pluridisciplinaires à dominante SVT (Chic Planet ${ }^{7}$ ), qui demandent cependant à être adaptées aux progressions dans la discipline. Enfin, les enseignants peuvent avoir recours aux éditions allemandes dont la médiathèque du Centre de formation aux enseignements bilingues (CFEB, Guebwiller) de l'IUFM d'Alsace offre un large répertoire en consultation. La situation est de ce fait paradoxale : en toute logique, l'édition régionale privilégie les outils interdisciplinaires, limite le stock disponible à un seul manuel par discipline. Mais utiliser des outils interdisciplinaires ou des manuels allemands exige une expérience pédagogique que les enseignants frais émoulus du CFEB 
n'ont pas encore. Ceci explique en partie que, malgré les publications disponibles, ils dénoncent la pénurie de matériel. équipes pour la production de dossiers dans les disciplines à enseigner en allemand (maths, histoire, SVT etc.) et en lecture. Actuellement, la tendance est à la publication de manuels (un manuel existant pour l'histoire en 4e, une grammaire bilingue annoncée pour 2010, un manuel de géographie et un manuel d'histoire en 3e pour 2009.)

\section{Questions ouvertes}

\section{1. Au niveau régional}

L'une des discussions récurrentes porte sur la définition de la langue régionale elle même et sur les liens entre la langue standard et les dialectes alémanique et francique parlés en Alsace et en Moselle. Dans les représentations des locuteurs, ce n'est pas l'allemand, mais le dialecte qui est la langue régionale. Certes, l'allemand n'a plus la présence qu'il avait encore - dans la presse, les cultes, l'édition - avant 1970 et n'a plus d'existence dans la vie sociale. Il ne peut pas être à lui seul la langue régionale, sans les dialectes. Une politique scolaire en faveur des dialectes est donc l'enjeu des classes bilingues (RUDIO, 2007). Une première réponse, mentionnée plus haut, a été apportée en classe de sixième.

Dans la formation elle - même, la place du dialecte est un enjeu de taille: dans les formations bilingues, on a pu évaluer la part de la dialectophonie aux deux tiers du public accueilli, mais les tabous sont loin d'être levés par rapport au dialecte. En effet, comme le constate Anemone GEIGER - JAILLET,

« les [futurs] enseignants, eux - mêmes, manquent d'assurance dans ce domaine et ne se sont pas interrogés sur les conclusions à tirer de leur propre biographie linguistique. La formation devrait donc les amener nettement plus à s'interroger et à réfléchir à ce sujet. » (GEIGER - JAILLET, 2006, p. 372, notre traduction)

Un autre débat porte sur la discontinuité relative entre la classe de 3e et le lycée. La seule voie de continuité pour les élèves ayant suivi à ce stade 12 années d'enseignement bilingue est la filière ABIBAC. Mais cette filière franco - allemande est une filière littéraire ( $6 \mathrm{~h}$ d'allemand et $4 \mathrm{~h}$ d'histoire en allemand) et sélective. Certes, des tentatives sont faites dans deux lycées sur la douzaine où fonctionne la filière pour en faire une suite logique des classes de 3e bilingues. L'académie de Strasbourg a compris le besoin de développer la place des disciplines scientifiques dans des sections européennes et d'y augmenter l'horaire d'enseignement en allemand pour assurer une véritable continuité avec le collège. Enfin, dans les lycées professionnels, qui disposent eux aussi de sections européennes en allemand, la tendance est à l'élargissement des stages en entreprises chez le voisin allemand ou suisse : des formules de validation existent déjà pour de tels stages. La même tendance se dessine dans les lycées d'enseignement généraux et technologique, 
mais avec une durée maximale de séjour de 6 mois, alors que des établissements suisses proposent déjà des séjours d'immersion d'un an (ELMIGER, 2008). Le chantier est donc loin d'être clos. moins en Alsace, grâce au concours spécial des professeurs des écoles de langue régionale, celui des professeurs de lycées et des collèges ne l'est pas, tout simplement, parce que les académies de Strasbourg et de Nancy - Metz sont privées d'un CAPES bivalent. Mais, même pour les PE, des difficultés subsistent : ainsi les postes mis au concours en Moselle, de l'ordre de 1 ou de 0 par an, sont notoirement insuffisants et dissuasifs. En Alsace, le vivier d'étudiants bons germanophones est inférieur d'une dizaine aux emplois mis au concours: le nombre des admissibles, ayant le niveau des compétences linguistiques exigées, se situe autour de la quarantaine seulement (sessions observées : 2005 à 2008). Si les $\mathrm{PE}$ alsaciens de langue régionale sont effectivement affectés à des classes bilingues, les arrêtés de titularisation ne reconnaissent pas leur spécificité et favorisent ainsi les mutations hors de la voie bilingue. Une réforme du concours permettant d'ouvrir davantage l'accès du concours à des germanophones de langue maternelle, pourrait contribuer à l'augmentation des viviers de recrutement.

Nos régions n'ont, soit pas de politique linguistique du tout - c'est le cas de la Moselle soit une politique linguistique de compromis. Elles ne se donnent pas tous les moyens de leur politique. Pour pallier le manque de professeurs bilingues dans le second degré, pourquoi ne pas proposer des bourses d'études dès le DEUG, comme on l'a fait pour les Professeurs des écoles? Imaginer de telles politiques incitatives, proposer aux universités de créer des viviers dès le DEUG, augmenter la place de la langue régionale dans le concours de $\mathrm{PE}$ et obtenir du ministère un CAPES bivalent pour la langue régionale d'Alsace et des Pays mosellans constituent des priorités. Les propositions récentes de l'association Lehrer vont dans ce sens (Association Lehrer, 2008)

\section{2. Au niveau national}

31 Dans le cadre de la réforme actuelle des concours de recrutement, le maintien et le développement des concours spéciaux de langue régionale et des CAPES bivalents constituent bien évidemment des nécessités absolues. Mais une telle évolution est freinée par l'incapacité des acteurs de langues régionales à déposer des propositions communes. Chaque région a ses orientations spécifiques, parfois irréductibles. Certes, cette situation a toujours existé et reflète la diversité de notre pays. Mais, jusqu'ici, elle n'a pas empêché des évolutions communes ni des adaptations régionales du cadre commun, par exemple celui de 1995 (circulaire DARCOS) ou celui formé en 2001 par un ensemble cohérent de textes sur l'enseignement des langues régionales. On a vu, ainsi, l'échec d'une tentative de concertation émanant, en 2008, d'une initiative du Centre de formation en breton (CFEB) de Saint - Brieuc, coordonnée par Stephan MOAL. Les associations d'enseignants n'ont pas de position commune. De plus, l'élément institutionnel fédérateur des langues régionales ne fonctionne plus non plus. 
32

mosellans, reconnue dans les textes de 2001 a failli disparaître dans les nouveaux textes. Dans l'indifférence générale, le ministère a fait préparer de nouveaux textes de programmes - destinés à remplacer ceux de 2003 - dans toutes les langues, sauf la nôtre. De plus, le ministère a publié des programmes de langue régionale pour le collège, dans toutes les langues, sauf celles d'Alsace et de Moselle. Nous avons été mis devant le fait accompli : seule une réaction immédiate de l'Association Lehrer auprès du Ministère, soutenue par les Collectivités territoriales, a permis le rétablissement in fine d'une partie de ces programmes dans l'arrêté complémentaire du 27 décembre 2007 déjà cité et le maintien de la reconnaissance de la langue régionale dans ses composantes dialectales et standard. Mais ni les programmes de langue pour le collège, ni ceux pour l'enseignement bilingue n'ont été publiés.

\section{Conclusion}

Une loi comme celle annoncée pour 2009 sur les langues de France ne peut offrir d'intérêt que si elle dessine le cadre dans lequel toutes les mesures attendues vont être effectivement - prises: lever les difficultés du suivi de la scolarité bilingue au lycée, compléter les modes de recrutement et de titularisation, ajuster le contenu des concours, garantir la reconnaissance de toutes les langues régionales, surtout la plus menacée, celle d'Alsace et des Pays mosellans et, enfin, inciter à la signature de conventions entre les autorités académiques et les collectivités territoriales, là où elles font cruellement défaut.

\section{BIBLIOGRAPHIE}

Académie de Strasbourg : site http://www.ac-strasbourg.fr/

ARDOUIN, Frank. (2009). Les sites biculturels mosellans, Revue Info-Lehrer, 2008/09, n²2.

Association Lehrer, association des instituteurs et des professeurs pour l'enseignement bilingue dans les académies de Strasbourg et de Nancy - Metz (2008a) : Ich möchte Lehrer werden - Devenir enseignant bilingue. - Association Lehrer : 13a, rue de la Schwang 67340 Weinbourg.

Association Lehrer (2008b) : Info - Lehrer 2008/09 n² et http://associationlehrer.blog.de/

ELMIGER, Daniel. (2008). La maturité bilingue en Suisse. La mise en œuvre variée d'une innovation de la politique éducative. Berne : Secrétariat d'État à l'éducation et à la recherche (S.E.R.) 62 p.

GEIGER - JAILLET, Anémone. (2006). Sprachattitüden zukünftiger bilingualer Lehrkräfte im Elsass, Nouveaux Cahiers d'allemand, 24e année, n4 (décembre), pp. 349 - 377.

GEIGER - JAILLET, Anémone / MORGEN Daniel. (2006). Le bilinguisme alsacien au service des enfants et des familles. Mise en perspective historique, linguistique et pédagogique de l'enseignement bilingue en Alsace, Revue Les Langues modernes, ${ }^{\circ} 2$, juin, pp. 78 - 89. 
HUCK, Dominique. (2006). L'enseignement de l'allemand à l'école primaire en Alsace entre 1945 et 1985, Revue d'Alsace, pp 337 - 406.

HUCK, Dominique. (2008). Langues et locuteurs en Alsace : approches sociolinguistiques, approches de politique linguistique, Revue d'Alsace, pp 345 - 354.

LERALU, Claudine, LICHAU, Isabelle. (2000). Pays basque français : un enseignement bilingue équilibré - Actualité de l'enseignement bilingue, Le Français dans le Monde, janvier, pp 63 à 68 .

MARCHAL, Anita. (2008). L'enseignement bilingue paritaire dans l'académie de Strasbourg, dans Haataja, Kim, Curriculum Linguae - Diversité linguistique à travers l'intégration, l'innovation et les échanges, Tampere, pp 223 - 228.

OLCA (Office pour la langue et la culture d'Alsace) - Région Alsace (2009) : Le bilinguisme en Alsace. (s.d. - février 2009).

RUDIO, Yves (2007). Le dialecte alsacien, parent pauvre de l'enseignement bilingue en Alsace, Nouveaux Cahiers d'allemand, 25e année, n², pp. $151-158$.

\section{NOTES}

1. Section = filière ouverte pour l'ensemble de la scolarité de collège ou de lycée. $(4 \mathrm{e}-3 \mathrm{e}$ ou seconde - 1re - T comptées pour une section).

2. Les effectifs des classes associatives $\mathrm{ABCM}$ sont déjà totalisés avec les classes sous contrat et les classes hors contrat (les deux types de classes existent dans les écoles $\mathrm{ABCM}$ ).

3. (H.Géo : 51 - Maths : 50 - E.P.S : 15 - S.V.T : 8 - Éducation musicale : 3 - Sciences physique : 3 et Technologie : 2 ).

4. Les informations qui suivent ont été rédigées et communiquées par Frank ARDOUIN, Inspecteur de l'Éducation nationale, directeur du Centre transfrontalier de Saint - Avold (Moselle) en novembre 2008.

5. http://www3.ac-nancy-metz.fr/iamoselle/centretransfrontalier/

6. Les données pour la Moselle ne sont pas disponibles.

7. Le CRDP est obligé d'arrêter cette publication pour raisons financières.

8. «Die Lehrkräfte sind jedoch selbst verunsichert, haben ihre eigene Sprachenbiographie vielfach nicht bewusst reflektiert und müssten während der Ausbildung viel stärker mit dem Thema konfrontiert werden."

\section{RÉSUMÉS}

Avant d'aborder le sujet proposé, les auteurs, engagés de longue date dans les évolutions linguistiques de leur région, décryptent la relation des Alsaciens et Mosellans germanophones avec leurs langues. Puis leur texte analyse les divergences des politiques linguistiques et pédagogiques : très présentes dans la construction du dispositif alsacien, les collectivités et autorités scolaires ont pris beaucoup plus de recul par rapport au dispositif mosellan. Une loi sur les langues régionales ne résoudra pas les problèmes, mais elle pourra avoir un effet dynamique en faveur de l'engagement des unes et des autres. 
Im folgenden Beitrag wird die Frage der Sprachen im Elsaß und in Lothringen angesprochen. Das Konzept der Regionalsprache, das seit Jahren für die alemannischen und fränkischen Dialekte und die deutsche Hochsprache benützt wird, bekundet eher die Unsicherheit der Bevölkerung aber auch die Ansichten der Behörden : lieber französisch als Dialekt, aber wenn's nicht anders geht, dann lieber noch Dialekt als Deutsch! Kann ein neues Gesetz diese Schwierigkeiten beseitigen? Sicher nicht, aber immerhin kann es das Wesen der Sprachen klar legen und alle Hindernisse, die hie und da auftauchen, hauptsächlich im fränkischsprachigen Teil Lothringens, lösen und eine richtige, kohärente Politik in der Schule aufbauen helfen. Folgender Text schildert den heutigen Stand des allgemeinen, extensiven sowohl als des immersiven Sprachunterrichts in allen Schularten und beschreibt die fördernden Maßnahmen.

\section{INDEX}

Schlüsselwörter : alemannisch, deutsch, eintauchenunterricht, Elsass, französisch, lehrerausbildung, Lothringen, mundarten, regionalsprachen, sprachenunterricht

Mots-clés : Alsace, enseignement, formation des maîtres, langue régionale, Pays Mosellans

\section{AUTEURS}

\section{DANIEL MORGEN}

Association Lehrer des instituteurs et professeurs pour l'enseignement bilingue dans les académies de Strasbourg et de Nancy - Metz

\section{ARMAND ZIMMER}

Association Lehrer des instituteurs et professeurs pour l'enseignement bilingue dans les académies de Strasbourg et de Nancy - Metz 